\title{
Los think tanks en el gabinete: una exploración del caso chileno (2006-2014)
}

\author{
Alejandro Olivares L.* \\ Bastián González-Bustamante** \\ Javiera Meneses*** \\ Matías Rodríguez****
}

\begin{abstract}
Resumen
Este artículo analiza los principales factores que permiten el acceso a los gabinetes de Michelle Bachelet (2006-2010) y Sebastián Piñera (2010-2014) en Chile. Se evalúa con especial atención la influencia que presenta la participación en un think tank para acceder al gabinete. Se trabaja con una base de datos de 102 casos que corresponden a 90 agentes que fueron nombrados ministros en el período estudiado. Se presenta una descripción del grupo y un Análisis Comparado Cualitativo (QCA). Se evidencia que la pertenencia a think tanks es un factor relevante para los nombramientos en ministerios políticos y económicos durante ambos gobiernos.
\end{abstract}

Palabras clave: Gabinete - think tanks - élite política - QCA - Chile.

\begin{abstract}
This paper analyses the main factors that allow access to Michelle Bachelet's cabinet (2006-2010) and to Sebastián Piñera's cabinet (2010-2014) in Chile, with focus on the influence of participation in think tanks to access the cabinet. We work with a database of 102 cases corresponding to 90 people who were appointed ministers in the mentioned period. We present a description of the group, and a Qualitative Comparative Analysis (QCA). The results find that think tank membership is a relevant factor for appointments in political and economic ministries during both governments.
\end{abstract}

Keywords: Cabinet - think tanks - political elite - QCA - Chile.

* Doctor (c) en Ciencias Sociales, Universidad de Chile. Docente de la Escuela de Gobierno y Gestión Pública, Universidad de Chile. Correo electrónico: aleolivares@iap.uchile.cl

** Magíster en Ciencia Política, Universidad de Chile. Investigador asociado del Centro de Análisis e Investigación Política (CAIP). Adscrito al Centro de Investigación Sociedad y Políticas Públicas (CISPO), Universidad de Los Lagos. Docente de la Escuela de Gobierno y Gestión Pública, Universidad de Chile. Correo electrónico: bastiangb@ug.uchile.cl

*** Licenciada en Ciencias Políticas y Gubernamentales, Universidad de Chile. Correo electrónico: javiera.meneses@gmail.com

**** Licenciado en Ciencias Políticas y Gubernamentales, Universidad de Chile. Correo electrónico: mati.rodriguez. cabrera@gmail.com 


\section{INTRODUCCIÓN}

El estudio de los gabinetes, en el contexto de los sistemas presidenciales, es un fenómeno relativamente reciente. El estudiar los gabinetes y a los ministros permite observar cómo se reparte el poder dentro de las coaliciones gobernantes y cómo se distribuye el poder dentro del sistema político (Avendaño y Dávila, 2012). Desde mediados de los 90, numerosos trabajos han dado cuenta de estos fenómenos estudiando la relación entre el presidente y los partidos para conformar y mantener los gabinetes (Alemán y Tsebelis, 2012; Altman, 2000; Amorim Neto, 1998, 2006; Cox y Morgenstern, 2001). Otros trabajos analizan la composición de los gabinetes en diferentes casos (Amorim Neto y Samuels, 2010). También existen estudios sobre los efectos de los gabinetes en el sistema político (Altman y Castiglioni, 2009; Amorim Neto y Borsani, 2004; Martínez-Gallardo, 2012), o la posibilidad de que en los casos donde no está permitida la reelección los gabinetes otorguen la oportunidad de contar con algún sucesor cercano al presidente en ejercicio (Olivares, 2012).

Entre las diferentes posibilidades para estudiar los gabinetes, el estudio de las carreras ministeriales ha tenido un importante desarrollo -tanto teórico como empírico- en Europa, posterior a la tercera ola de las democratizaciones. Para América Latina, el desarrollo de estudios sobre las carreras de los ministros es escaso, y en general existe la tendencia a estudiarlas como parte de una temática más general, vinculada al estudio de las élites políticas (González-Bustamante, 2013a). Según Dávila (2011), algunos autores se centran en definir las trayectorias académicas de quienes están en el poder; un ejemplo es el trabajo de Camp (1985). Otros, como Teichman (2001), relacionan las trayectorias académicas con las ideológicas en particular.

Para el caso chileno existen trabajos que intentan vincular capitales de los ministros con ministerios en los cuales se han desempeñado (Dávila et al., 2013), y sobre las lógicas de distribución de ministerios (Avendaño y Dávila, 2012; Fernández y Rivera, 2013). Del mismo modo, existen trabajos que identifican algunas características claves para acceder a la élite política gubernamental: género (masculino), la edad (haber nacido entre 1940 y 1950), los recursos técnicos referidos a poseer estudios de posgrado, y la vinculación a un think tank ligado a la coalición gobernante (González-Bustamante, 2013b). Un reciente trabajo de Olivares et al. (2014) demuestra que para los ministros de la Concertación haber pertenecido a think tanks vinculados a dicha coalición aumenta sus posibilidades de estar en el gabinete durante todo el gobierno.

La vinculación a un centro de pensamiento o think tank suele ser considerada como una credencial de experticia político-académica importante, ya que estas instituciones son uno de los ejemplos de tecnificación de la política y las políticas. Por esta razón, resulta una perspectiva de trabajo interesante el identificar las vinculaciones entre capitales de ministros, think tanks y gabinete. En esa línea, este artículo busca contribuir, de manera exploratoria, al conocimiento sobre la carrera de los ministros en Chile. Se busca identificar hasta qué punto algunas estructuras formales e informales del sistema político sirven como fuentes de reclutamiento para que los presidentes elijan ministros. En este contexto, nos preguntamos si la participación en un centro de estudio o think tank es una condición necesaria para 
ser nombrado ministro. Para responder esta pregunta se realiza un análisis descriptivo y un Qualitative Comparative Analysis (QCA) con los integrantes de los gabinetes de los gobiernos de Michelle Bachelet (2006-2010) y Sebastián Piñera (2010-2014).

El presente documento se divide en cuatro secciones. Primero se realiza una revisión bibliográfica con los aportes más importantes sobre formación de gabinetes, el fenómeno de acumulación de capitales técnicos y políticos, y el estudio de los think tanks con especial énfasis en su conceptualización y clasificación. La segunda sección corresponde a un apartado metodológico que entrega detalles sobre el diseño y las técnicas de análisis. El tercer apartado corresponde a la presentación de resultados; allí se expone un análisis descriptivo y los resultados del QCA. Para finalizar se presentan las principales conclusiones de la investigación.

\section{GABINETES Y THINK TANKS}

\subsection{Gabinetes y ministros}

La formación de coaliciones es clave para entender la conformación de los gabinetes. El perfil del ministro, la distribución de ministros por partidos, o los motivos de salida de los ministros, dependerán en gran medida de las políticas de coalición. Se ha demostrado que un gabinete partidista limitará el campo de acción del presidente, mientras que uno con mayor cantidad de no militantes y/o tecnócratas facilitará al presidente su capacidad de maniobra, ya que su preocupación por los costos políticos es baja (Altman y Castiglioni, 2009). La distribución partidaria de los ministros depende en gran medida de la relación entre los poderes ejecutivo y legislativo. Si el ejecutivo quiere gobernar con el apoyo del legislativo tenderá a nombrar un gabinete más partidista, mientras que si quiere gobernar sin el congreso, tenderá a nombrar a un gabinete menos partidista (Amorim Neto, 1998, 2006). Por otra parte, si el presidente se enfrenta con un congreso con múltiples atribuciones, o muy profesionalizado, necesitará un gabinete que le permita entrar en la negociación legislativa y conseguir finalmente victorias políticas para su gestión (Alemán y Tsebelis, 2012).

Independiente de los factores estructurales recién señalados, los ministros pueden ser agrupados en tres tipos de agentes: tecnócratas, technopols y dirigentes de partidos (Joignant, 2011; véase también González-Bustamante, 2013a) ${ }^{1}$. Los tecnócratas son de dos tipos. El primero, Ilamado tecnócrata pragmático, puede ser políticamente independiente y posee un capital a base de una expertise determinada. El segundo tipo de tecnócrata es el político, el que cuenta con habilidades técnicas pero a la vez desarrolla un capital político mínimo, generalmente expresado en una militancia partidista. El tecnócrata político no se caracteriza precisamente por alcanzar posiciones relevantes en la dirigencia partidaria.

1 Esto se complementa con las diferentes especies de capitales políticos que distinguen Joignant et al. (2012): (a) capital familiar; (b) capital universitario; (c) capital político militante; (d) capital político oligárquico; (e) capital tecnocrático pragmático; (f) capital tecnocrático político; (g) capital tecnopolítico; (h) capital de notoriedad; e (i) capital carismático. 
Por otra parte, los technopols presentan una convergencia simultánea de dos especies de capital. Por una parte gozan de un capital cultural expresado en altos recursos técnicos, lo que se manifiesta en niveles educaciones muy elevados, muchas veces a base de posgrados realizados fuera de Chile, particularmente en Estados Unidos o en ciertos lugares de Europa. Pero además se trata de individuos que disponen de una amplia red de contactos y un capital político importante, el que se relaciona con la ocupación de posiciones de poder en partidos políticos antes de alcanzar nombramientos ministeriales. Todo esto les permite diferenciarse de los tecnócratas políticos.

Finalmente, el tercer tipo de agente que caracteriza Joignant (2011) se refiere a los dirigentes de partido, quienes también ostentan dos tipos de capital y es posible encontrar dos subtipos de agentes. Por una parte el capital militante lo poseen quienes han pasado toda una vida al alero de un partido político, sin haber accedido a posiciones relevantes de poder dentro del mismo. Por otra parte, el capital político oligárquico lo poseen quienes ocupan cargos relevantes al interior de los partidos, sobre todo a nivel nacional, en la línea weberiana clásica del político profesional (Alcántara, 2012). El capital político oligárquico puede ser heredado por intermedio de dinastías familiares o tener un carácter social, es decir, ser adquirido mediante redes (Joignant, 2011; véase también González-Bustamante, 2013a).

\subsection{El rol de los think tanks}

Una de las redes informales que poseen mayor importancia en la formación de capitales de los agentes que Ilegan a altos cargos en la gestión pública son los think tanks o centros de estudio o pensamiento. La interdependencia entre el poder político y los think tanks ha sido uno de los fenómenos de mayor relevancia en el campo de la administración y la política (Molina e Iglesias, 2005). Esta interdependencia se ve reforzada en el caso chileno, pues los centros de pensamiento tuvieron un rol protagónico en el reclutamiento político durante la transición a fines de la década de 1980 y comienzos de 1990; esto implicó un movimiento desde estos centros al poder ejecutivo (Delamaza, 2013). Esto se relaciona con la expansión de la tecnopolítica, fenómeno entendido como el aumento de la invocación de legitimidad técnica en posiciones de privilegio (Centeno y Silva, 1998; Delamaza, 2011).

El desarrollo de los centros de pensamiento da cuenta de la necesidad de la élite gubernamental de establecer relaciones con actores complementarios en un país donde los partidos políticos siguen siendo hegemónicos (Flisfisch et al., 2013). El concepto de think tank ha estado ligado a planes de evaluación y estrategia militar desde finales de la primera mitad del siglo XX, sin embargo, con el tiempo el término pasa a asociarse a procesos de formulación de políticas públicas en temas políticos, económicos y sociales (GonzálezBustamante, 2013b; McGann y Weaver, 2002; Navarrete y Gómez Amigo, 2011).

En América Latina, estos centros han tenido un rol relevante fijando la agenda intelectual, entrenando a futuros líderes gubernamentales, y promoviendo políticas públicas (McGann y Weaver, 2002; Tompkins, 2007). El origen y el desarrollo de estos centros de pensamiento en Chile han respondido al contexto sociohistórico del país. Es posible identificar como un 
primer hito relevante el surgimiento de los "Centros Académicos Privados" (CAP) (Brunner, 1985). En este marco, una de las primeras experiencias se remonta al año 1957 por iniciativa de la Organización de las Naciones Unidas para la Educación, la Ciencia y la Cultura (UNESCO). Se trata de la Facultad Latinoamericana de Ciencias Sociales (FLACSO Chile). Posteriormente surgen una serie de centros de estudios que también buscaron desarrollar espacios de análisis y debates relevantes sobre la realidad chilena (Flisfisch et al., 2013). Otros autores, como Delamaza (2013), también fechan el surgimiento de los primeros centros de pensamiento durante la década de 1960, principalmente con el apoyo de la Iglesia Católica y la cooperación internacional, particularmente norteamericana. Varios de estos centros tenían como fin respaldar el proyecto político del gobierno de Eduardo Frei Montalva (1964-1970).

Por su parte, Cociña y Toro (2009) identifican tres grandes momentos relacionados con el "ciclo de vida" de los think tanks en Chile. El primer momento es durante el régimen militar y la transición (1973-1990), en el cual proliferaron distintas organizaciones gracias a la ayuda internacional. Un segundo momento se da durante el retorno a la democracia y el proceso de consolidación (1990-2000), el cual destaca por la migración de los agentes desde estos espacios al gobierno, lo que significó la desaparición de varios centros. Finalmente, identifican, desde el año 2000 en adelante, una tercera etapa relacionada con el surgimiento de tendencias más transversales y la reconfiguración de instituciones de larga data. Cabe señalar que a 2013 Chile cuenta con 42 think tanks activos (McGann, 2014).

En definitiva, los think tanks remiten a la existencia de una determinada expertise que los posiciona y visibiliza. En el caso chileno es posible advertir el funcionamiento de centros de estudio o grupos de expertos al margen de los procesos políticos formales, pero que nutren y dan forma a los programas políticos a base de un conocimiento que no es el conocimiento teórico propio de la academia, sino más bien un subproducto dirigido a los tomadores de decisiones (Gárate, 2008; Pinilla, 2012). Estos centros de pensamiento, dependiendo del tipo al cual pertenezcan en función de sus características particulares, pueden ser un gran espacio de desarrollo para los tecnócratas políticos y technopols.

Efectivamente es posible distinguir entre diferentes tipos de think tanks. Una propuesta de clasificación es la de Flisfisch et al. (2013) que apunta a una ubicación ideológica dentro del espectro político. También resulta interesante la propuesta de Pinilla (2012) que los clasifica según su funcionalidad siguiendo la lógica de los trabajos de McGann y Weaver (2002), Gárate (2008) y Navarrete y Gómez Amigo (2011). La propuesta distingue entre los siguientes tipos de think tanks: (a) centros de apoyo (advocacy think tanks), los que otorgan un respaldo experto a la promoción de ideas y valores de los distintos actores involucrados en la definición de asuntos públicos; (b) centros partidarios (party think tanks), una variante del anterior que implica un vínculo mucho más directo con un partido político específico; (c) centros de gestión (contract think tanks), donde se encuentran las instituciones que tienen un perfil similar al de consultoras privadas; estas instituciones no tienen pretensiones de incidir en la agenda pública, pero buscan influenciar en niveles específicos en el contexto de los procesos de formulación de políticas públicas; y (d) centros académicos o "universidades 
sin estudiantes" (academic think tanks), los que se focalizan en investigación y publicación, y además se caracterizan por una fuerte confianza en académicos e investigadores.

En general, los centros ligados a partidos políticos tienden a alejarse de la noción clásica del concepto que entiende a los think tanks como centros autónomos que generan conocimiento manteniendo distancia de partidos políticos y universidades (McGann y Weaver, 2002; véase también González-Bustamante, 2013b; Navarrete y Gómez Amigo, 2011). En definitiva, la clasificación de un centro de pensamiento se relaciona con el concepto espacio de los think tanks de Medvetz (2012), el cual sería un subespacio de producción del conocimiento que surge de la intersección entre el campo político, económico, académico o cultural, y mediático. En este sentido, si se considera que diferentes estructuras de capital, siguiendo a Bourdieu (1980, 1997), "rinden" de forma distinta dependiendo del campo específico², lo mismo aplicaría para los think tanks: ciertas estructuras de capital son más funcionales dependiendo del tipo de centro de pensamiento (e.g., los centros académicos privilegian más el capital cultural que los centros partidarios).

\section{MÉTODO}

\subsection{Evaluando la participación en think tanks como determinante del nombramiento ministerial}

El objetivo de esta investigación es determinar en qué grado, si es que existe, la pertenencia a un think tank es un mecanismo de acceso a puestos ministeriales. Para esto se realiza en primer lugar un análisis estadístico descriptivo de un conjunto de datos que está compuesto por todos los ministros de los gobiernos de Michelle Bachelet (2006-2010) y de Sebastián Piñera (2010-2014). Luego se realiza un Qualitative Comparative Analysis (QCA) ${ }^{3}$ para determinar cuáles son las características más importantes a la hora de la selección para la conformación del gabinete. El uso del QCA se debe a su capacidad para explicar la relación existente entre las diferentes combinaciones de condiciones y un resultado determinado (Wagemann, 2012), poniendo a prueba las variables independientes para determinar la condición de causalidad que poseen respecto de la variable dependiente (González-Bustamante, 2013b, 2013c). Cabe señalar que el QCA se basa en álgebra booleana para maximizar el número de comparaciones en una lógica determinista, no probabilística (Rihoux, 2006; Rihoux y Marx, 2013).

La utilización de esta técnica permite establecer si es que la pertenencia a un centro de estudios es un factor determinante en el proceso de conformación de gabinetes, y cuáles son las otras variables que también se erigen como determinantes. Específicamente se utiliza el conjunto clásico o crisp-set QCA (csQCA) basado en variables binarias o dicotómicas. En una primera instancia se presentan resultados de un análisis de condiciones necesarias

2 El capital cultural, por ejemplo, tiene un buen rendimiento en el campo académico-intelectual, sin embargo se puede suponer que no tiene un rendimiento tan bueno -en términos comparativos- en el campo político.

3 Para más detalle revisar Ragin $(1987,2000,2006,2013)$. 
para el nombramiento en determinados tipos de ministerio en los distintos gobiernos; para estos efectos se evalúan distintas disyunciones inclusivas. Posteriormente, para analizar las condiciones suficientes y vislumbrar las conjunciones de variables predominantes para nombramientos en distintos tipos de ministerios, se trabaja con el algoritmo Quine-McCluskey. Esta técnica arroja tres tipos de solución: parsimoniosa, intermedia y compleja. Se trabaja con los resultados de la solución parsimoniosa que ofrece la respuesta teórica más simple y asume que las configuraciones $\sin \operatorname{casos}^{4}$ hubiesen producido el resultado de interés. Esto implica la simplificación de los casos contrafactuales.

\subsection{Características de los datos utilizados}

La base de datos ha sido construida según información recolectada desde fuentes secundarias de acceso público. Está conformada por todos los ministros entre los años 2006 y 2014, es decir, los ministros de los gobiernos de Michelle Bachelet (2006-2010) y Sebastián Piñera (2010-2014). Esto da un total de 102 casos (90 personas) que se distribuyen en 48 nombramientos de ministros ${ }^{5}$ en el primer gobierno de Bachelet (45 personas); y 54 nombramientos en el gobierno de Piñera (45 personas).

\subsection{Diseño de investigación y variables}

Las variables seleccionadas tras un análisis teórico son género, edad, partido político, capital cultural, capital político, y pertenencia a un think tank. El capital cultural se basa en la descripción de recursos técnicos de Joignant (2011) y González-Bustamante (2013b), que obedece a la definición clásica de capital cultural institucionalizado de Bourdieu (1980). El capital cultural en su estado institucionalizado entrega credenciales que tienden a consagrar de forma casi permanente la posición de los individuos en el espacio social, el cual aglutina agentes de acuerdo con su estructura de capital, es decir, el peso relativo de distintos tipos de capital y su volumen total (Bourdieu, 1980, 1997). Esto se ha medido con la posesión de al menos un posgrado.

El capital político se ha medido en función de carreras políticas. Se ha considerado el ejercicio anterior al nombramiento ministerial en algún cargo político relevante, sea este de elección popular o designación. También se ha considerado verificar una trayectoria como asesor electoral. Esta distinción significa comprender el capital político de una forma "negada", similar al concepto de capital simbólico de Bourdieu (1980). Esto implica una forma de acumulación que no se expresa necesariamente en la acumulación de capital económico, pero sí potencia redes de aliados y relaciones mediante un entramado de deudas de honor y vínculos basados en lealtad. La forma "negada" adquiere una acumulación

4 Se trata de aquellas configuraciones de variables que son lógicamente posibles, pero empíricamente no se verifican casos.

5 En el análisis se incluye a Felipe Harboe, quien asumió el cargo de ministro del interior suplente durante una semana en enero de 2008. 
volviéndose un crédito en el sentido más amplio -y literal- de la palabra, crédito que aplica específicamente al campo político.

Como variable dependiente se ha elegido el tipo de ministerio, esto con el fin de analizar la condición de causalidad que las demás variables establecen con el nombramiento en uno u otro tipo de ministerio. Se ha utilizado la distinción realizada por Joignant (2011), dejando por un lado a los ministerios de carácter político o económico y, por otro, a las carteras de carácter social.

TABLA 1

Resumen de variables y dicotomización

\begin{tabular}{|c|c|c|c|}
\hline \multicolumn{2}{|c|}{ VARIABLE Y SÍMBOLO } & MEDICIÓN & $\begin{array}{c}\text { VALOR } \\
\text { BINARIO }\end{array}$ \\
\hline \multicolumn{4}{|c|}{ VARIABLES INDEPENDIENTES } \\
\hline$x_{1}$ & Género [GEN] & $\begin{array}{l}\text { Masculino } \\
\text { Femenino }\end{array}$ & $\begin{array}{l}1 \\
0\end{array}$ \\
\hline$x_{2}$ & Capital cultural [CC] & $\begin{array}{l}\text { Poseer al menos un posgrado } \\
\text { No poseer un posgrado }\end{array}$ & $\begin{array}{l}1 \\
0\end{array}$ \\
\hline$x_{3}$ & Partido político [PP] & $\begin{array}{l}\text { Militar en un partido político } \\
\text { No militar en un partido político }\end{array}$ & $\begin{array}{l}1 \\
0\end{array}$ \\
\hline$X_{4}$ & Capital político [CP] & $\begin{array}{l}\text { Haber ocupado antes del nombramiento un cargo de confianza } \\
\text { política (ministro, subsecretario, director de empresa pública), } \\
\text { de representación democrática (alcalde, concejal, diputado } \\
\text { o senador) y/o participación como asesor electoral (miembro } \\
\text { de grupo programático, jefe de campaña, asesor de campaña) } \\
\text { No haber ocupado antes del nombramiento como ministro } \\
\text { cargos de confianza política, de representación democrática } \\
\text { y/o participación como asesor electoral }\end{array}$ & 1 \\
\hline$X_{5}$ & $\begin{array}{l}\text { Participación en think } \\
\text { tanks [TT] }\end{array}$ & $\begin{array}{l}\text { Pertenecer a un think tank antes del nombramiento } \\
\text { No pertenecer a think tanks antes del nombramiento }\end{array}$ & $\begin{array}{l}1 \\
0\end{array}$ \\
\hline \multicolumn{4}{|c|}{ VARIABLES DEPENDIENTES } \\
\hline$Y_{1}$ & $\begin{array}{l}\text { Tipo de ministerio en } \\
\text { gobierno de Bachelet }\end{array}$ & $\begin{array}{l}\text { Ministerio político o económico } \\
\text { Ministerio social }\end{array}$ & $\begin{array}{l}1 \\
0\end{array}$ \\
\hline $\mathrm{Y}_{2}$ & $\begin{array}{l}\text { Tipo de ministerio en } \\
\text { gobierno de Piñera }\end{array}$ & $\begin{array}{l}\text { Ministerio político o económico } \\
\text { Ministerio social }\end{array}$ & $\begin{array}{l}1 \\
0\end{array}$ \\
\hline
\end{tabular}

Nota: En el apartado de resultados se utilizan distintos símbolos adicionales como negación $(\neg X)$, disyunción inclusiva $\left(X_{1}+X_{2}\right)$ y conjunción $\left(X_{1} * X_{2}\right)$.

Fuente: Elaboración propia. 


\section{RESULTADOS}

\subsection{Análisis descriptivo}

Género [GEN]. Como es esperable, existe una mayor presencia masculina en los dos períodos gubernamentales analizados: $68,6 \%$ hombres y $31,4 \%$ mujeres. Sin embargo, al analizar ambos períodos por separado se puede apreciar una marcada diferencia: en el gobierno de Michelle Bachelet (2006-2010) la presencia femenina alcanzó un 41,7\%, justificada principalmente por la política de equidad de género al comienzo de su mandato; en el gobierno de Sebastián Piñera (2010-2014), por otra parte, la incorporación de mujeres en el gabinete alcanzó solo un $22,2 \%$.

Posesión de capital cultural [CC]. Se aprecia que al menos un 56,9\% del conjunto posee un posgrado. No se distinguen grandes diferencias entre ambos períodos estudiados: 58,3\% para los ministros del gobierno de Michelle Bachelet; 55,6\% para los ministros del gobierno de Sebastián Piñera.

Militancia en partido político [PP]. Es en este aspecto donde se aprecia la diferencia más importante entre ambos gobiernos. En este punto se hace evidente la conocida intención de Sebastián Piñera de formar un gabinete técnico, pues solo un 51,9\% de todos sus ministros presentan militancia política. De forma opuesta, durante el gobierno de Michelle Bachelet un 91,7\% de los ministros eran parte de alguno de los partidos de la coalición de gobierno. Esta diferencia deja en evidencia los distintos criterios que se utilizaron a la hora de conformar gabinetes en cada período.

Posesión de capital político [CP]. Un 66,7\% de los casos habían ejercido un cargo político relevante de forma previa. Es importante recordar que se consideran cargos de primera línea en el ejecutivo y legislativo, pero también la participación relevante como asesor electoral. La cifra se eleva en el gobierno de Sebastián Piñera a un 70,4\%, mientras que en el gobierno de la Concertación baja al 62,5\%. Ahora bien, si solo se consideran los cargos de primera línea, la situación cambia drásticamente: menos del $20 \%$ en el gobierno de Piñera; cerca del $40 \%$ en el gobierno de Bachelet. Esto quiere decir que el capital político de los ministros de Piñera fue adquirido principalmente mediante asesorías electorales y no ocupando cargos políticos relevantes.

Participación en think tanks [TT]. Al menos un 27,5\% del conjunto había participado en algún centro de pensamiento antes de su nombramiento ministerial. La cifra se eleva al $39,6 \%$ en los ministros del gobierno de Bachelet, y baja a un 16,7\% al evaluar los ministros del gobierno de Piñera.

Tipos de ministerios. Al analizar la frecuencia de la variable dependiente se advierte que en un $57,8 \%$ del conjunto se verifica nombramiento en algún ministerio político o económico. En ambos períodos las cifras mantienen esta tendencia. En el período de Michelle Bachelet los nombramientos en ministerios políticos y económicos $\left(\mathrm{Y}_{1}\right)$ ascienden al 60,4\%, mientras que en el período de Sebastián Piñera $\left(\mathrm{Y}_{2}\right)$ la cifra alcanza 
el 55,6\%. Evidentemente estas cifras están condicionadas por la estructura formal del gabinete chileno.

\subsection{Qualitative Comparative Analysis (QCA)}

\subsubsection{Análisis de condiciones necesarias para nombramiento en ministerios políticos y económicos (csQCA)}

Un primer análisis de condiciones necesarias realizado con cada conjunto de datos arroja que las tres mejores disyunciones inclusivas para el gabinete de Bachelet $\left(\mathrm{n}_{1}=48\right)$ son: $\left(X_{2}+X_{3}\right)$ y $\left(X_{3}+X_{5}\right)$ con una cobertura de 0,62 y una consistencia de 1,00; y $\left(X_{1}+X_{5}\right)$ con una cobertura de 0,74 y una consistencia de 0,90.

Por otra parte, para el gabinete de Piñera $\left(n_{2}=54\right)$, estas combinaciones quedan de la siguiente forma: $\left(X_{1}+X_{3}\right)$ y $\left(X_{1}+X_{4}\right)$ con una cobertura de 0,59 y una consistencia de 0,97 ; $y\left(X_{1}+X_{5}\right)$ con una cobertura de 0,64 y una consistencia de 0,93 .

En resumen, para el caso del gobierno de Bachelet emergen como condiciones necesarias para el nombramiento ministerial en carteras políticas y económicas la posesión de capital cultural, la militancia en un partido político y la participación en think tanks. Para el gobierno de Piñera, por otra parte, las condiciones necesarias para el nombramiento son ser hombre, la militancia en un partido político, poseer capital político y participar en centros de pensamiento.

En ambos casos la participación en think tanks es una condición que puede ser considerada necesaria (no así suficiente) para el nombramiento en carteras políticas o económicas.

\subsubsection{Análisis con algoritmo Quine-McCluskey para nombramientos por tipo de ministerio en el gobierno de Michelle Bachelet (2006-2010)}

Es posible identificar seis configuraciones causales que en su conjunto conforman una solución parsimoniosa para explicar la integración de un ministerio político o económico durante el gobierno de Michelle Bachelet $\left(\mathrm{Y}_{1}\right)$ (Tabla 2). La solución cubre solo un $45 \%$ de la muestra, sin embargo explica todos los casos que verifican la variable dependiente, es decir, es completamente consistente.

La solución para las configuraciones con mayor cobertura para los nombramientos en carteras políticas y económicas se puede expresar de la siguiente forma:

$$
\mathrm{Y}_{1}=(\neg \mathrm{CC} * \mathrm{TT}) *(\mathrm{GEN}+\mathrm{CP})
$$

En esta síntesis emergen como condiciones para integrar un ministerio político o económico el no poseer un alto capital cultural institucionalizado, pero sí haber pertenecido a algún think tank, pudiendo esto combinarse con ser hombre o con poseer capital político antes del nombramiento. 
TABLA 2

Configuraciones causales conducentes a ocupar ministerios políticos y económicos $\left(\mathrm{Y}_{1}\right)$ y ministerios sociales $\left(\neg Y_{1}\right)$ durante el gobierno de Michelle Bachelet (2006-2010)

\begin{tabular}{|c|c|c|c|}
\hline \multicolumn{4}{|c|}{$\mathrm{Y}_{1}=\mathrm{F}(\mathrm{GEN}, \mathrm{CC}, \mathrm{PP}, \mathrm{CP}, \mathrm{TT})$} \\
\hline CONFIGURACIÓN CAUSAL & $\begin{array}{c}\text { TASA DE } \\
\text { COBERTURA } \\
\text { TOTAL }\end{array}$ & $\begin{array}{c}\text { TASA DE } \\
\text { COBERTURA } \\
\text { EXCLUSIVA }\end{array}$ & $\begin{array}{c}\text { ÍNDICE DE } \\
\text { CONSISTENCIA }\end{array}$ \\
\hline GEN * $\neg \mathrm{CC} * \mathrm{TT}$ & 0,17 & 0,00 & 1,00 \\
\hline$\neg \mathrm{CC} * \mathrm{CP} * \mathrm{TT}$ & 0,17 & 0,00 & 1,00 \\
\hline $\mathrm{GEN} * \neg \mathrm{CP}$ & 0,14 & 0,14 & 1,00 \\
\hline $\mathrm{CC} * \neg \mathrm{PP}$ & 0,10 & 0,00 & 1,00 \\
\hline$\neg \mathrm{PP} * \mathrm{TT}$ & 0,10 & 0,00 & 1,00 \\
\hline$\neg \mathrm{GEN} * \neg \mathrm{CC} * \mathrm{CP}$ & 0,04 & 0,04 & 1,00 \\
\hline Tasa de cobertura de la solución & 0,45 & & \\
\hline Tasa de consistencia de la solución & 1,00 & & \\
\hline \multicolumn{4}{|c|}{$\neg \mathrm{Y} 1=\mathrm{F}(\mathrm{GEN}, \mathrm{CP}, \mathrm{PP}, \mathrm{CP}, \mathrm{TT})$} \\
\hline CONFIGURACIÓN CAUSAL & $\begin{array}{c}\text { TASA DE } \\
\text { COBERTURA } \\
\text { TOTAL }\end{array}$ & $\begin{array}{c}\text { TASA DE } \\
\text { COBERTURA } \\
\text { EXCLUSIVA }\end{array}$ & $\begin{array}{c}\text { ÍNDICE DE } \\
\text { CONSISTENCIA }\end{array}$ \\
\hline$\neg \mathrm{GEN} * \neg \mathrm{CP} * \neg \mathrm{TT}$ & 0,42 & 0,16 & 0,89 \\
\hline$\neg \mathrm{GEN} * \neg \mathrm{CC}^{*} \neg \mathrm{CP}$ & 0,32 & 0,00 & 0,86 \\
\hline$\neg \mathrm{GEN} * \neg \mathrm{CC} * \mathrm{TT}$ & 0,05 & 0,00 & 1,00 \\
\hline$\neg \mathrm{CC} * \neg \mathrm{CP} * \mathrm{TT}$ & 0,05 & 0,00 & 1,00 \\
\hline Tasa de cobertura de la solución & 0,47 & & \\
\hline Tasa de consistencia de la solución & 0,90 & & \\
\hline
\end{tabular}

Nota: Solución parsimoniosa a base del algoritmo Quine-McCluskey.

Fuente: Elaboración propia.

En cuanto a la integración de ministerios sociales durante el gobierno de Bachelet $\left(\neg Y_{1}\right)$, el algoritmo arroja cuatro configuraciones causales para la solución parsimoniosa. La 
cobertura de la solución es un poco más alta que la anterior y un 90\% de los casos verifica la variable dependiente. La solución de las configuraciones con mayor cobertura se puede expresar de la siguiente forma:

$$
\neg \mathrm{Y}_{1}=\neg \mathrm{GEN} *(\neg \mathrm{CC}+\neg \mathrm{CP})
$$

La síntesis evidencia que las condiciones para integrar ministerios sociales privilegian el ser mujer, lo cual se puede combinar con no poseer un alto capital cultural o no poseer capital político antes del nombramiento. En este sentido, no es posible apreciar patrones claros para los nombramientos en ministerios sociales, sin embargo, la evidencia sugiere que es el tipo de ministerios al cual las mujeres pudieron acceder en mayor medida durante el gobierno de Bachelet. En definitiva, la vinculación a centros de pensamiento resulta relevante para los nombramientos en ministerios políticos y económicos.

\subsubsection{Análisis con algoritmo Quine-McCluskey para nombramientos por tipo de ministerio en el gobierno de Sebastián Piñera (2010-2014)}

Al revisar la conformación del gabinete durante el gobierno de Sebastián Piñera $\left(\mathrm{Y}_{2}\right)$ se identifican seis configuraciones causales para nombramientos en ministerios políticos y económicos (Tabla 3). La solución tiene una cobertura baja, sin embargo, es completamente consistente.

La síntesis de las configuraciones con mayor cobertura para los nombramientos en carteras políticas y económicas durante el gobierno de Sebastián Piñera se puede expresar de la siguiente forma:

$$
\mathrm{Y}_{2}=(\mathrm{GEN} * \neg \mathrm{CC}) *(\neg \mathrm{PP}+\neg \mathrm{CP}+\mathrm{TT})
$$

La síntesis muestra que durante el gobierno de Piñera el ser hombre y no poseer un alto capital cultural fue fundamental para integrar ministerios políticos o económicos. Esto se combina con la ausencia de militancia política, la falta de capital político antes del nombramiento, o la vinculación a think tanks.

Por otra parte, respecto de la integración de ministerios sociales, la solución presenta una cobertura muy baja. Las configuraciones causales que destacan se pueden sintetizar en la posesión de capital cultural acompañada de una militancia política. Esto es interesante, pues la evidencia sugiere que en el gobierno de Sebastián Piñera las variables políticas (militancia y capital político) estarían más asociadas a la integración de los ministerios de carácter social que a los ministerios políticos y económicos.

\section{CONCLUSIONES}

La participación en think tanks es una variable que resulta determinante en los nombramientos en ministerios políticos y económicos durante ambos gobiernos, sin embargo, es un factor 
TABLA 3

Configuraciones causales conducentes a ocupar ministerios políticos y económicos $\left(\mathrm{Y}_{2}\right)$ y ministerios sociales $\left(\neg Y_{2}\right)$ durante el gobierno de Sebastián Piñera (2010-2014)

\begin{tabular}{|c|c|c|c|}
\hline \multicolumn{4}{|c|}{$\mathrm{Y}_{2}=\mathrm{F}(\mathrm{GEN}, \mathrm{CC}, \mathrm{PP}, \mathrm{CP}, \mathrm{TT})$} \\
\hline CONFIGURACIÓN CAUSAL & $\begin{array}{c}\text { TASA DE } \\
\text { COBERTURA } \\
\text { TOTAL }\end{array}$ & $\begin{array}{c}\text { TASA DE } \\
\text { COBERTURA } \\
\text { EXCLUSIVA }\end{array}$ & $\begin{array}{c}\text { ÍNDICE DE } \\
\text { CONSISTENCIA }\end{array}$ \\
\hline GEN * $\neg \mathrm{CC} * \neg \mathrm{PP}$ & 0,17 & 0,07 & 1,00 \\
\hline $\mathrm{GEN} * \neg \mathrm{CC} * \neg \mathrm{CP}$ & 0,13 & 0,03 & 1,00 \\
\hline $\mathrm{GEN} * \neg \mathrm{CC} * \mathrm{TT}$ & 0,07 & 0,07 & 1,00 \\
\hline$\neg \mathrm{PP} * \mathrm{CP} * \mathrm{TT}$ & 0,07 & 0,07 & 1,00 \\
\hline$\neg \mathrm{GEN} * \mathrm{CC} * \mathrm{PP} * \mathrm{CP}$ & 0,03 & 0,00 & 1,00 \\
\hline$\neg \mathrm{GEN} * \mathrm{CC} * \mathrm{TT}$ & 0,03 & 0,00 & 1,00 \\
\hline Tasa de cobertura de la solución & 0,37 & & \\
\hline Tasa de consistencia de la solución & 1,00 & & \\
\hline \multicolumn{4}{|c|}{$\neg Y_{2}=\mathrm{F}(\mathrm{GEN}, \mathrm{CP}, \mathrm{PP}, \mathrm{CP}, \mathrm{TT})$} \\
\hline CONFIGURACIÓN CAUSAL & $\begin{array}{c}\text { TASA DE } \\
\text { COBERTURA } \\
\text { TOTAL }\end{array}$ & $\begin{array}{c}\text { TASA DE } \\
\text { COBERTURA } \\
\text { EXCLUSIVA }\end{array}$ & $\begin{array}{c}\text { ÍNDICE } \\
\text { DE } \\
\text { CONSISTENCIA }\end{array}$ \\
\hline$\neg \mathrm{GEN} * \mathrm{CC}^{*} \neg \mathrm{TT}$ & 0,13 & 0,13 & 1,00 \\
\hline $\mathrm{GEN} * \mathrm{CC} * \mathrm{PP} * \mathrm{TT}$ & 0,08 & 0,08 & 1,00 \\
\hline$\neg \mathrm{CP} * \mathrm{TT}$ & 0,04 & 0,04 & 1,00 \\
\hline$\neg \mathrm{GEN} * \neg \mathrm{CC} * \mathrm{TT}$ & 0,04 & 0,04 & 1,00 \\
\hline Tasa de cobertura de la solución & 0,29 & & \\
\hline Tasa de consistencia de la solución & 1,00 & & \\
\hline
\end{tabular}

Nota: Solución parsimoniosa a base de algoritmo Quine-McCluskey.

Fuente: Elaboración propia. 
más relevante durante el gobierno de Michelle Bachelet (2006-2010). Si bien solo un 27,5\% de los ministros en el período 2006-2014 había participado en un think tank antes de su nombramiento, esta cifra se eleva a un $39,6 \%$ para el gobierno de Bachelet y baja a un $16,7 \%$ para el gobierno de Piñera. Esta simple comparación ya ofrece un panorama de las condiciones en las cuales la pertenencia a un centro de pensamiento se vuelve un factor determinante para acceder al gabinete.

A pesar de que los análisis de condiciones necesarias sobre los distintos conjuntos de datos muestran que la participación en think tanks es una variable que puede ser considerada necesaria (no suficiente) para el nombramiento ministerial en carteras políticas o económicas, los análisis con el algoritmo Quine-McCluskey muestran resultados más específicos para cada administración analizada.

Durante el gobierno de Michelle Bachelet la pertenencia a think tanks es una variable muy importante para el nombramiento en ministerios políticos y económicos. La importancia de los centros de pensamiento se conjuga con la ausencia de alto capital cultural institucionalizado, es decir, credenciales académicas relevantes y, alternativamente, con alguna de las siguientes variables: ser hombre o poseer capital político. Sobre el capital político, es importante señalar que ha sido entendido como la ocupación de un cargo político relevante o posiciones como asesor electoral antes del nombramiento ministerial. Para los nombramientos en ministerios sociales no resulta relevante la vinculación a centros de pensamiento.

Por otra parte, durante el gobierno de Sebastián Piñera, la participación en think tanks resulta relevante para el nombramiento en ministerios políticos y económicos, aunque no con la misma preponderancia que en la administración de Bachelet. En estos ministerios se detecta un fuerte predominio masculino, el cual ya se advierte en el análisis descriptivo, pues la presencia femenina en el gabinete de Piñera alcanza solo un 22,2\%, lejos del 41,7\% del gobierno de Bachelet. Este predominio masculino está acompañado de una ausencia de un capital cultural institucionalizado relevante, y de forma alternativa con las siguientes variables: ausencia de militancia política, falta de capital político, o participación en think tanks antes del nombramiento.

Para los nombramientos en ministerios sociales durante el gobierno de Piñera, los patrones son algo erráticos, sin embargo se detecta que la posesión de capital cultural y una militancia política son factores importantes. Esto último es Ilamativo, pues sugiere que en el gobierno de Piñera las variables políticas estarían más asociadas a la integración de los ministerios de carácter social y no a los ministerios políticos o económicos. Esta tendencia es bastante distinta a las prácticas de la Concertación entre 1990 y 2010 . En este contexto, es importante tener en cuenta la intención de Sebastián Piñera por formar un gabinete técnico con muchos independientes y outsiders, sin embargo los datos evidencian que el gabinete de Piñera no presentó mejores credenciales académicas que el de Bachelet: un 58,3\% de los ministros de Bachelet verifica alto capital cultural versus el 55,6\% de los ministros de Piñera. También es relevante recalcar que el capital político de los ministros de Piñera no está asociado a cargos de primera línea en el ejecutivo o legislativo, sino más bien a cargos relacionados con campañas electorales. 
Es importante considerar que los análisis se han realizado sobre el número de casos y no el número de agentes. Las variables independientes para los casos de las personas que recibieron varios nombramientos fueron construidas respecto del primer nombramiento. También es importante tener en cuenta que las coberturas de los análisis con el algoritmo Quine-McCluskey para el gobierno de Piñera son relativamente bajas, en particular la cobertura del análisis de nombramientos en carteras sociales.

Como este es un estudio exploratorio, es conveniente mirar bajo ese prisma los resultados y evaluar posteriores investigaciones que incorporen nuevas variables que reflejen de mejor forma las trayectorias políticas de los agentes, o que impliquen operacionalizaciones alternativas de las variables utilizadas. En esta línea, considerar otros factores, en especial para analizar el gabinete de Sebastián Piñera, quizás permitiría explicar algunos elementos que en esta investigación no quedan totalmente claros. En este sentido, es importante considerar que las estructuras de capital de los individuos están intrínsecamente ligadas al espacio social y al campo específico en que estos agentes se desenvuelven, en este caso el campo político. Esto implica que los factores que explican el caso concertacionista no necesariamente explican bien lo que sucede en la derecha. Quizás en este punto sería interesante evaluar variables relativas a prácticas religiosas, vinculaciones a grupos empresariales, entre otros factores más constitutivos del ethos de la derecha chilena.

A pesar de las limitaciones mencionadas, los hallazgos de este trabajo respecto de la importancia de los think tanks para nombramientos en ministerios políticos y económicos durante ambos gobiernos, pero especialmente para el gobierno de Bachelet, constituyen resultados relevantes que van en la línea de estudios sobre los factores de acceso y permanencia de la élite gubernamental chilena que muestran la importancia de los centros de pensamiento durante los gobiernos concertacionistas (González-Bustamante, 2013b). Esto se vincula directamente a la interdependencia entre el poder político y los think tanks que señalan Molina e Iglesias (2005), y al rol que han tenido estos centros en América Latina respecto de la fijación de agenda y del entrenamiento de líderes políticos (McGann y Weaver, 2002; Tompkins, 2007). Todo esto se ha visto fuertemente reforzado en Chile por la existencia de una élite democrática que se convirtió en una élite tecnocrática durante la transición, situación que implicó un reclutamiento político donde los think tanks tuvieron un rol bastante importante (Delamaza, 2011, 2013). Esta situación se relaciona, tal como se ha señalado, con la expansión de la tecnopolítica, situación que ha dado pie para que tecnócratas y technopols sean figuras importantes en los gabinetes.

La evidencia de este trabajo indica que los think tanks fueron parte importante del entorno concertacionista, sirviendo incluso como instancias de reclutamiento político. $\mathrm{Si}$ bien ocurre lo mismo en la derecha, el fenómeno parece no darse con la misma magnitud. Lo anterior no solo sucede porque puedan existir otros factores que sean más relevantes para la cultura política de la derecha chilena, sino que también ocurre porque poseen menos centros de estudios partidarios, además varios en realidad presentan mayor cercanía con el campo académico. Ahora bien, varios ministros de Piñera que no tenían vínculos con think tanks se integraron a alguno cuando dejaron el gabinete. En este marco, es importante 
considerar que la vinculación con un centro de estudios en Chile, sin importar su tipo, tiende a asociarse a la posesión de credenciales académicas importantes, pero los hallazgos de esta investigación muestran que no necesariamente es así: muchos agentes efectivamente fueron parte de think tanks y eso les ayudó para ingresar al gabinete, sin embargo no poseían un alto capital cultural.

\section{BIBLIOGRAFÍA}

Alcántara, M. (2012): El oficio de político, Tecnos, Madrid.

Alemán, E. y G. Tsebelis (2012): “Partidos políticos y coaliciones de gobierno en las Américas", Política, Revista de Ciencia Política, 50 (2), pp. 5-32.

Altman, D. (2000): "The politics of coalition formation and survival in multiparty presidential democracies. The case of Uruguay, 1989-1999", Party Politics, 6 (3), pp. 259-283.

Altman, D. y R. Castiglioni (2009): “Gabinetes ministeriales y reformas estructurales en América Latina, 1985-2000", Revista Uruguaya de Ciencia Política, 18 (1), pp. 15-39.

Amorim Neto, O. (1998): "Cabinet formation in presidential regimes: an analysis of 10 Latin American Countries", ponencia presentada en el XXI Congreso LASA, Chicago, Estados Unidos.

(2006): "The presidential calculus: executive policy making and cabinet formation in the Americas", Comparative Political Studies, 39 (4), pp. 415-440.

Amorim Neto, O. y H. Borsani (2004): "Presidents and cabinets: the political determinants of fiscal behavior in Latin America", Studies in Comparative International Development, 39 (1), pp. 3-27.

Amorim Neto, O. y D. Samuels (2010): "Democratic regimes and cabinet politics: a global perspective", Revista Ibero-Americana de Estudios Legislativos, 1 (1), pp. 10-23.

Avendaño, O. y M. Dávila (2012): “Rotación ministerial y estabilidad coalicional en Chile 1990-2010", Política, Revista de Ciencia Política, 50 (2), pp. 87-108.

Bourdieu, P. (1980): Le sens pratique, Les Éditions de Minuit, Paris.

(1997): Capital cultural, escuela y espacio social, Siglo XXI Editores, México D.F.

Brunner, J.J. (1985): “La participación de los Centros Académicos Privados", Estudios Públicos, 19, pp. 1-12.

Camp, R.A. (1985): "The political technocrat in Mexico and the survival of the political system", Latin American Research Review, 20 (1), pp. 97-118.

Centeno, M.A. y P. Silva (1998): "The politics of expertise in Latin America: introduction", en M.A. Centeno y P. Silva (eds.): The politics of expertise in Latin America, St. Martin's Press, New York, pp. 1-12.

Cociña, M. y S. Toro (2009): "Los think tanks y su rol en la arena política chilena”, en E. Mendizábal y K. Sample (eds.): Dime a quién escuchas... Think tanks y partidos políticos en América Latina, IDEA Internacional \& ODI, Perú, pp. 98-126.

Cox, G.W. y S. Morgenstern (2001): "Latin America's reactive assemblies and proactive presidents", Comparative Politics, 33 (2), pp. 171-189.

Dávila, M. (2011): Governing together: the Concertación administrations in Chile (1990-2009), University of North Carolina-Chapel Hill, Chapel Hill, NC. 
Dávila, M., A. Olivares L. y O. Avendaño (2013): “Los gabinetes de la Concertación en Chile (19902010)", América Latina Hoy, 64, pp. 67-94.

Delamaza, G. (2011): "Elitismo democrático, líderes civiles y tecnopolítica en la reconfiguración de las élites políticas", en A. Joignant y P. Güell (eds.): Notables, tecnócratas y mandarines: elementos de sociología de las élites en Chile (1990-2010), Ediciones Universidad Diego Portales, Santiago de Chile, pp. 77-108.

(2013): "De la élite civil a la élite política. Reproducción del poder en contextos de democratización", Polis, Revista Latinoamericana, 12 (36), pp. 67-100.

Fernández, M.A y E. Rivera (2013): “Instituciones informales, coaliciones y gabinetes en el presidencialismo chileno", Política, Revista de Ciencia Política, 51 (1), pp. 155-184.

Flisfisch, A., M. Prieto y A. Siebert (2013): "Potenciando universidades y think tanks en América Latina: El caso de Chile", FLACSO Chile, Santiago de Chile, disponible en http://www.flacsochile.org/ wp-content/uploads/2014/04/Potenciando-universidades-y-think-tanks-en-Am\%C3\%A9ricaLatina-El-caso-de-Chile1.pdf

Gárate, M. (2008): “Think Tanks y Centros de Estudio. Los nuevos mecanismos de influencia política en el Chile post-autoritario", en M. de Cea, P. Díaz y G. Kerneur (eds.): Chile: ¿ De país modelado a país modelo? Una mirada sobre la política y la economía, LOM Ediciones, Santiago de Chile, pp. 67-85.

González-Bustamante, B. (2013a): “El estudio de las élites en Chile: aproximaciones conceptuales y metodológicas", Revista Intersticios Sociales, 6, pp. 1-20.

(2013b): "Factores de acceso y permanencia de la élite política gubernamental en Chile (1990-2010)", Política, Revista de Ciencia Política, 51 (1), pp. 119-153.

(2013c): “Trayectorias y patrones de carreras políticas de ministros en Chile (1990-2010)", ponencia presentada en el XI Congreso Argentino de Ciencia Política, Paraná, Argentina.

Joignant, A. (2011): “Tecnócratas, technopols y dirigentes de partido: tipos de agentes y especies de capital en las élites gubernamentales de la Concertación (1990-2010)", en A. Joignant y P. Güell (eds.): Notables, tecnócratas y mandarines: Elementos de sociología de las élites en Chile (1990-2010), Ediciones Universidad Diego Portales, Santiago de Chile, pp. 49-76.

Joignant, A., L. Perelló y J. Torres (2012): “Las fuentes del poder político. Fundamentos para una teoría del capital político a partir de la evidencia chilena", ponencia presentada en el XXII Congreso Mundial de Ciencia Política, Madrid, España.

McGann, J.G. (2014): 2013 global go to think tank index report, University of Pennsylvania, Think Tanks and Civil Societies Program, Philadelphia, disponible en http://gotothinktank.com/ the-2013-global-go-to-think-tank-index-ggttti/

McGann, J.G. y R.K. Weaver (2002): "Think tanks and civil societies in a time of change", en J.G. McGann y R.K. Weaver (eds.): Think tanks and civil societies: catalysts for ideas and action, Transaction Publishers, New Brunswick, pp. 1-36.

Martínez-Gallardo, C. (2012): "Out of the cabinet: what drives defections from the government in presidential systems?", Comparative Political Studies, 45 (1), pp. 62-90.

Medvetz, T. (2012): Think tanks in America, The University of Chicago Press, Chicago.

Molina, D. y M. Iglesias (2005): "Poder e ideas: el papel de los think tanks en el diseño y ejecución de la política exterior estadounidense", Revista Electrónica de Estudios Internacionales, 10, pp. 1-28. 
Navarrete, B. y G. Gómez Amigo (2011): "Los centros de pensamiento (think tanks) en la Democracia Cristiana. ¿Más política que políticas?", en M. Mella (ed.): Extraños en la noche: intelectuales y usos políticos del conocimiento durante la transición chilena, RIL Editores, Santiago de Chile, pp. 241-282.

Olivares L., A. (2012): "Los ministros precandidatos presidenciales en los gobiernos de la Concertación", ponencia presentada en el IV Congreso Uruguayo de Ciencia Política, Montevideo, Uruguay.

Olivares L., A., J. Baeza F. y M. Dávila (2014): “Los gabinetes ministeriales en la democracia chilena post-1990: Un caso de estabilidad, continuidad histórica y negociación inter-partidaria", manuscrito inédito, Instituto de Asuntos Públicos, Universidad de Chile, Santiago de Chile.

Pinilla, J.P. (2012): "Think Tanks, saber experto y formación de agenda política en el Chile actual", Polis, Revista Latinoamericana, 11 (32), pp. 119-140.

Ragin, C.C. (1987): The comparative method: moving beyond qualitative and quantative strategias, University of California Press, Berkeley, CA. (2000): Fuzzy-set social science, University of Chicago Press, Chicago.

(2006): "Set relations in social research: evaluating their consistency and coverage", Political Analysis, 14 (3), pp. 291-310.

(2013): "New directions in the logic of social inquiry", Political Research Quarterly, 66 (1), pp. 171-174.

Rihoux, B. (2006): "Qualitative Comparative Analysis (QCA) and related systematic comparative methods. Recent advances and remaining challenges for social science research", International Sociology, 21 (5), pp. 679-706.

Rihoux, B. y A. Marx (2013): "Qualitative Comparative Analysis at 25: state of play and agenda", Political Research Quarterly, 66 (1), pp. 167-171.

Teichman, J.A. (2001): The politics of freeing markets in Latin America: Chile, Argentina, and Mexico, The University of North Carolina Press, North Carolina.

Tompkins, E.K. (2007): "Think tanks and public policy research institutes", Behavioral \& Social Sciences Librarian, 26 (2), pp. 11-27.

Wagemann, C. (2012): “QQué hay de nuevo en el método comparado?: QCA y el análisis de los conjuntos difusos", Revista Mexicana de Análisis Político y Administración Pública, 1 (1), pp. 51-75. 